\title{
Contemporary Evolution in CRISPR-CAS9 System Technology and their Uses in Treatment of Human Common Diseases: A Review
}

\author{
Zainab Sajid Mohammed ${ }^{1}$, Wijdan Rajh Hamza Al-Kraity ${ }^{2}$ \\ ${ }^{1}$ Ph.D. in Histophysiology, Lec. Dr. at College of Pharmacy, Ahl Al-bait University, ${ }^{2}$ Ph.D. in Medical Physiology, \\ Lec. Dr. at Department Medical Laboratories Techniques, Al-Toosi University College
}

\begin{abstract}
Gene therapy has proven its potential in treatment of several human diseases. Most recent method in a long line of the genome-editing-techniques is called "A Clustered-Regularly-Interspaced-Short-PalindromicRepeat-associated-protein9" abbreviated as CRISPR-Cas9. The scientists and researcher have long sought to the control, and modification the DNA that consider "the code of life". The CRISPR-Cas9technology is offer very important improvements that differ about other gene-editing.technologies, it's uncomplicated to apply \& low-cost, also have comparatively high degree of the efficiency and precision . This technology uses a ".ribonucleic acid.(RNA)-guided. Deoxyribonucleic. acid-DNA-endonuclease.", Cas9, which induces double-strand breaks (DSBs) in the target site, the DSBs was repaired by the use of a variety of cellular DNA-repair systems leading to changes in target sites, that technology have proven to be effective to investigation, prevention, and treatment of diseases.
\end{abstract}

This review summarizes contemporary evolution in CRISPR - Cas9 system technology and their uses in treatment of common diseases.

Keywords: Genome editing, CRISPR/Cas 9 technology and Gene therapy.

\section{Introduction}

The.Genome-editing-technologies is included mutations caused by UV radiation and chemicals, the zinc-finger-nucleases (ZFNs), the DNA-recombinase mediated gene replacement, and the "transcriptionalactivator-like-effector-nuclease (TALEN)" mechanisms, it have contributed significantly to both basic and clinical developments in the biological research ${ }^{\mathbf{1 , 2}}$.

Scientists have reformed or change the genes by using radioactivity or chemical materials. These procedures of modifying produce random outcomes. The development of the technology of the recombinant-DNA

\footnotetext{
Corresponding Author:

Dr. Zainab Sajid Mohammed

Ph.D. in Histophysiology, Lec. Dr. at College of

Pharmacy, Ahl Al-bait University

e-mail: dr.zainab1988@gmail.com
}

in a year (1970s) permitted to the researchers with adding a new fragment of DNA addicted to genes structure, but introducing a precise gene or sequence within the genome persisted large theoretically inaccurate and challenging ${ }^{3}$. Gene editing can be used to many process such as insert, eliminate, or transform DNA in a genome.

The CRISPR-Cas9 technology is one of the geneediting-technology that longer provide the possibility of making significant improvements over other geneediting-technologies ${ }^{4}$. That technology was described, easy to ably, efficacy, speed, also inexpensive ${ }^{5}$. the CRISPR-Cas9 technology have proven, it provide Revolutionary developments in the diagnosis, treatment and prevention of several diseases ${ }^{6}$.

\section{Definition and description of CRISPR-Cas9 technology}

The CRISPR-Cas9technology is one of a gene editing-technologies, its uses mixture of the enzyme called "a nuclease-Cas9 enzyme " to facilitate cuts DNA, 
and (guide-RNA) a guide piece of the genetic material to indicate a position in the genome ${ }^{\mathbf{1 , 2}}$, Usually, the guide RNA has been target and bind to the special-DNAsequence, while an attached-Cas9enzyme works on cleave both DNA strands at that location, that slash could be used to inserts, removes, or edits the DNA-sequence, After that a slash is repaired and the changes have been integrated, (Show fig1). CRISPR/Cas9technology is derived from the adaptive immunity of Streptococcus pyogenes bacterium ${ }^{7}$, where Cas9-nuclease-enzyme was mediated antiphage action recognition to its mixture with clustered frequently interspaced to CRISPR-loci that is a short palindromic repeats. that loci is short consist of 30-40 bp repetitive sequences and also intercalates with the spacing sequences match a virus genome ${ }^{8}$. And a CRISPR-loci has been transcribed to the long RNA that consequently has been cleaved.via "CRISPR-associated endo-ribonucleases" Known a Cas9 to liberate small CRISPR-RNAs (crRNAs). The crRNAs after that in cooperation with tracrRNA form a Cas-RNA complex which recognize the target DNA and profits for cleaving it; in general, four strategies has been utilized for "double-strand break repair include geneknockout ordisruption, "gene deletion via NHEJ way", "gene correction", \& "gene knock-in/insertion via HDR way"9 .

The initial appliance of the CRISPR-Cas9 coordination acts correspondingly to the immune systems in macroorganisms. After a bacterial or archaeal cell infected by virus, the fragment of the DNA viral categorization is incorporated into the CRISPR region in the bacteria ${ }^{\mathbf{1 0}}$. Immune response to CRISPR-Cas9 and the presence of preexisting antibodies against Cas9 (a protein) could be a significant hurdle especially for in vivo gene editing. It is highly desirable to exercise more rigorous assessment of possible immunological responses to the microbial origin of the system ${ }^{\mathbf{1 1}}$. However, delivery of RNP complexes and codon optimization may hold the key to overcome such hurdles ${ }^{12}$. The CRISPR/Cas9 technology have been proven effective in establishing a gene $\mathrm{KO}$ or knockdout that known knockin in human cells and is predominantly valuable for editing stimulated pluripotent stem cells or (iPSCs.) ${ }^{9}$. Despite that advances, several the ethical, technical \& biological researches limiting about utilization that technology in analysis and treatment of common diseases.

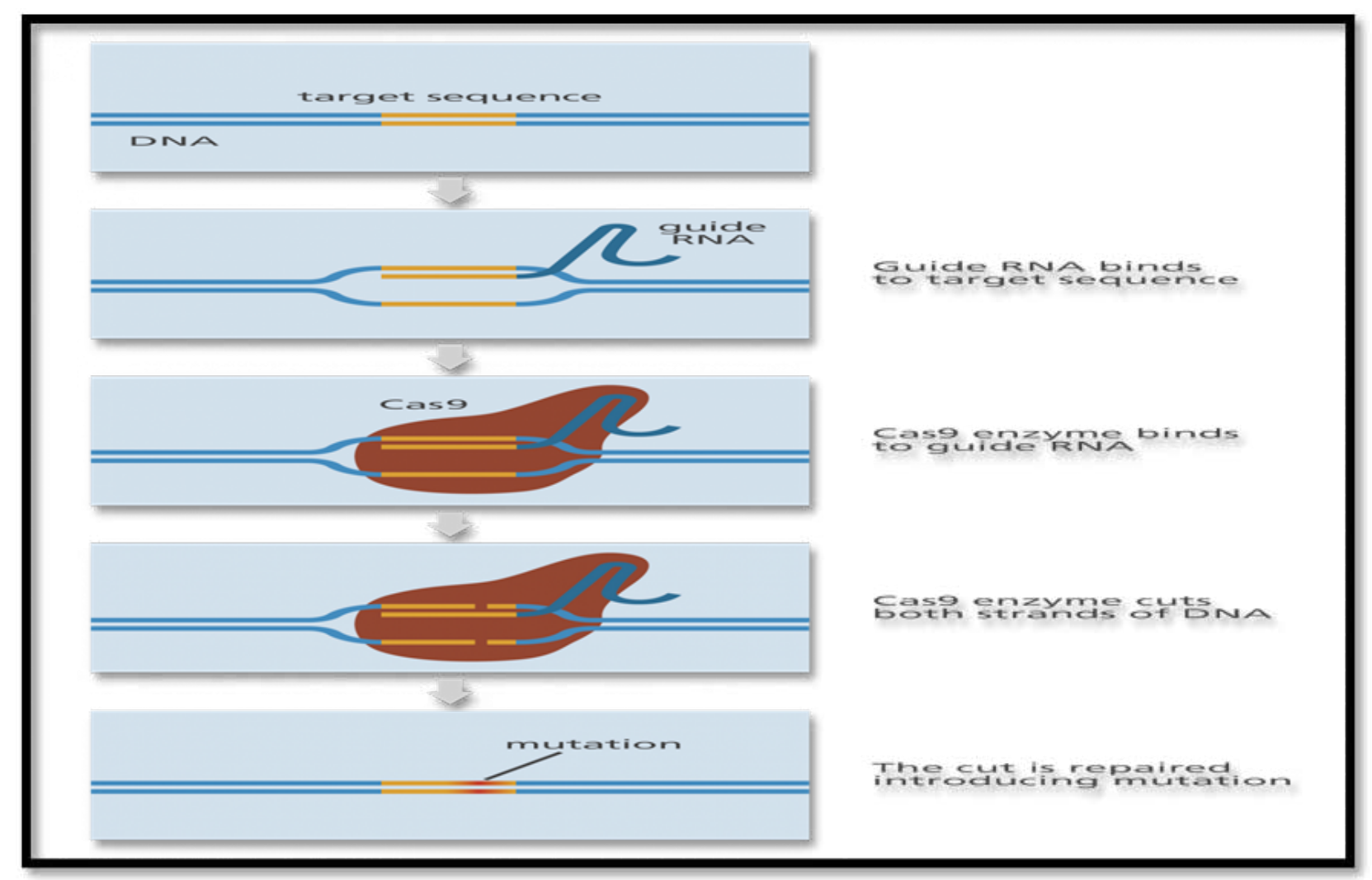

Fig. 1: "What Is CRISPR-Cas9? ${ }^{13}$," at http://www.yourgenome.org/facts/what-is-crispr-cas9 
Compensations or Advantage of CRISPR Cas9 technology: The genome editing system of CRISPR Cas-9 gives many benefits concluded the nuclease enzyme of zinc finger and transcription activator like effector nuclease in human iPSCs, and also stem cells of somatic origin .

Several synthesizing primers essential to be designated for expending this technique, since its specificity is associated only to ribo-nucleotide multifaceted development ${ }^{\mathbf{1 4}}$. Additional, these system is further economical, low cost for plasmid-mediated of that system. Another uses as the wildest presently obtain able technique of gene editing, it is using of these system can characteristically be achieved in present time ${ }^{\mathbf{1 5}}$. The CRISPR/Cas9 technology have been established to be valuable \& effective for an editing various human .cells 16-18. Recently, researchers have been reveal a higher editing capability of the transcription-activator-likeeffector-nuclease in human stem cells is more efficiency about $79 \%$, of using for editing human iPSCs ${ }^{19}$. Furthermore, CRISPR/Cas9 also utilizing to monitor DNA noncoding sequences for recognizing regulatory elements to understand how a genetic variation were associated with cause the many of human diseases ${ }^{\mathbf{2 0 - 2 1}}$; in this context, Fulco et al. recognized nine distal enhancers, and their target-gene via CRISPRi ${ }^{20}$, and Also the CRISPR/Cas9 have been utilize to set up DNA changes in the noncoding locations, and established a association between transcriptional function and intronic SNPs in PHACTR1gene in that location ${ }^{22}$. Where there is Another concern was raised with CRISPR/Cas9 technology relates to editing efficiency sgRNAs. induce Cas9-mediated DSB. by the side of favorite target position, a DSB. induces "DNA-repair by HDR. way", conversely, the alternative-"DNA-repair-mechanismNHEJ." be able to induce at low frequencies, giving rise to unpredictable events of diminutive insertions \& deletions ${ }^{23}$.

Application of CRISPR-Cas9 technology in treatment of the human common diseases: The previous scientists were able to create "alternativecell-models" via induced pluripotent stem cell (iPSC), for monitoring the molecular mechanisms of several diseases including cardiovascular disease, in the context of CRISPR/Cas9 system has been provide a straight forward mechanism for elucidating how cells mishandled via allowing with reversing of a causal mutation to the disease ${ }^{24-26}$. A Previous study of Wang et al. ${ }^{27}$. showed that persons with usually happening loss of thefunction mutation or what is called with "proprotein convertase subtilisin/kexin type 9" abbreviated as PCSK9.", they had lower of serum LDLC concentration, and thus edition of the "PCSK9.gene via CRISPR/Cas9technology" was a hopefully therapeutic board to avoidance of the cardiovascular disorder. While the study of Xie, et al. 28 indicated that utilized the CRISPR/Cas9technology in the postnatal mice to correct the mutation that cause to PRKAG2 cardiac syndrome, while the study of Ma et al. ${ }^{29}$ utilized a CRISPR/Cas9 technology for correction a pathogenic mutation, in the MYBPC3 gene that causes hypertrophic cardiomyopathy in human embryos.

1. The diabetes mellitus disease: The "California Institute for Regenerative Medicine (CIRM)" awarded a scholarship to the scientists. who work in the "Children's Hospital Los Angeles", they have been utilized the CRISPR-Cas9 technology for developing the .modified approach to treat a genetic variations of the diabetic disease (for example Type I diabetes mellitus) via replacement the insulin producing cells in diabetic patients, and scientists suppose that the technique may also ultimately suggest treatment for non autoimmune diabetic disease (for example Type II diabetes mellitus) $)^{\mathbf{3 0 , 3 1}}$. These study's findings were indicated that utilization of the "patient's own cells" reduced risks of the transplant rejection, also the patients wouldn't be dependent on a limited availability of external donors.

2. The respiratory diseases: The DF508 mutation have been recognized in (CFTR) is one respiratory disease and its recognized in nineteen percent of patients with a cystic fibrosis, and previous study indicated that improvement of the DF508mutation in pluripotent stem cell (iPCS) by the use of "CRISPR/ Cas9-mediated HDR" way to treat CFTR disease 32. In same the context, The "Alpha-1 Antitrypsin Deficiency (AATD)" that cause a type of respiratory disorder that corrected by utilization of CRISPR/ Cas9technology by way of the NHEJ. mediated gene-disruption \& the HDR. based precise genecorrection $^{33}$.

3. The hematologic diseases: Recently, the researchers have been utilized CRISPR/Cas9 technology to treatment the sickle cell disease (SCD) via two strategies : correction the mutation that cause sickle cell and stimulation the fetal hemoglobin (HbF) expression. In 2018 FDA established the submission of two bio-technology establishments-CRISPR 
and Vertex for an investigational management with gene therapy for (SCD).

The new Method of treatment by using system of CRISPR-Cas9 for modification of stem cells that separated from blood of patients and then rein-fused for production of $\mathrm{F} \mathrm{Hb}$. The advanced intensities of $\mathrm{F} \mathrm{Hb}$ are predictable to stabilize the pain that caused by the mutation of sickle cell ${ }^{\mathbf{3 4}}$ . In the gene correction technique have been used the hematopoietic stem cells that derivative from the SCD patients \& also corrected the Ex. Vivo. by the use of CRISPR/Cas9technology, after that transplanted into the SCD patients ${ }^{35}$

The study of Canver et al. ${ }^{36}$ indicated that the BCL11A." is a transcriptional controller which serve as a strong $\mathrm{Hb} F$ silencer \& suppress to expression of cglobin, So this is suppression of BCL11A. could be utilized to treatment both betathalassemia \& sickle cell anemia, while the study of Liu et al. ${ }^{37}$ indicated via utilization of the CRISPR/Cas9system, the beta-thalassemia causing mutation in patients derivative induced pluripotent stem cells (iPSCs) could be corrected \& after that transplants a corrected iPSCs into that same patient - Also by the CRISPR/Cas9-mediated HDR have utilized to correction a mutation in patient with haemophilia- $\mathrm{B}^{38}$. Majority a strict haemophilia-A were resulted via large-chromosomal inversions $(600$ or $140 \mathrm{~kb})$ within coagulation Factor VIII (F8), \& correction of those inversions in the patientderived-iPS cells were created by the transfer of RNP-complex of Cas9, \& a couple of sgRNAs for targeting two dissimilar locations for stimulating rein version of those mutations ${ }^{39}$.

5. The severe combined immunodeficiency disease: Currently, the study of Chang et al. ${ }^{40}$. indicated that using the "CRISPR/Cas9-mediated HDR approach" able to correction a point mutation at "the exon14 of JAK3gene" in patient-derived iPSC for treating severe combined immunodeficiency. Also the study of De Ravin et al. ${ }^{\mathbf{4 1}}$ indicated Patient-derived iPSC of "the X-linked chronic granulomatous-disease-XCGD- patients" that cause by a point mutation at the CYBB gene, could be corrected via "CRISPR/ Cas9-mediated HDR approach" and after that, transplants into the same patient. The Knockout of miR-155 is imperative pro-inflammatory controller in the rheumatoid arthritis disease,via CRISPR/ CAS9 technology have been revealed that the inhibition of pro-inflammatory-cytokine creation in "macrophage cell line" \& presented a optimistic therapeutic strategy to treatment of arthritis disease 42.

6. The neurological diseases: This is a comparatively new and more advanced technology of gene editing but there are several setbacks: first being the host genome itself, and Targets to modify may have enough sequence resemblance (similarity) with other part of genome that leads to unintended cuts in host genome. Thus, undesired mutations are generated, which may affect overall health and even survival of host organism adversely. In vivo delivery is another limiting factor. Crossing blood-brain barrier is an utmost challenge for components of this gene-editing technology $\mathbf{4 3 , 4 4}$.

There is two strategies have been done designed for gene therapy to treat the progressive degenerative neurological disorder, include: "deletion of pathogenic mutations and targeted gene correction". The CRISPR/Cas9technology could be ably for targeting deletion of the CAG repeats via the sgRNA/Cas9collection which flank this domain and creation a DSBs that consequently stimulate "nonhomologous end joining (NHEJ) process" ${ }^{45}$. And also the "(deletion of open-reading-frame of HTT)" could reduced mutant-huntingtin-masses ${ }^{46}$.Also the previous studies indicated that "Friedreich Ataxia and Amyotrophic lateral sclerosis (ALS)" were anther disease which have targeted via CRISPR/ Cas9technology 46-48.

The Malaria Disease: This is mosquito infected human and other animals, one of the greatest pervasive and mortal disease in the biosphere. Operative revision, decrease, or dismissal of the Anopheles mosquito is initial route for communication of the malaria can significantly condense expenditures also opened up original way for commercial occasions in domain's unfortunate nations.

CRISPR-Cas-9 permitted attitudes that including use of driving genes in a improved gene being differently approved to children. This powerfulness proposal a incomes by which all Anopheles mosquitos might be prepared sterile ${ }^{49}$. Also consequence in all young male 50. If effective, these methodologies will, considerably diminish or-even perhaps eliminate the populace being embattled. Additional method of CRISPR Cas9 permitted attitude pursues for making the Anopheles mosquito impervious to the malaria. 
7. The Resistance for antibiotics: Conferring to the center of disease control and prevention about two million of persons are infested yearly with microorganisms (bacteria) was appear resistance for antibiotics, also about thirty two thousands of individuals will decease or die every year and the consequence of such contaminations ${ }^{51}$. The new system of CRISPR/Cas9 had been revealed to successfully board and reduce of bacteria with different types, as well as the strains of bacteria that have resistance to antibiotics from a public of microorganisms. This accurate training permits removal of injurious of bacteria, but circumvents positive of bacteria . Furthermore, unlike oldfashioned or customy of antibiotics will be challenging for the bacteria to progression their resistances to the CRISPR -based antimicrobials since such as the resistance will probable destroys defense of the bacteria, permit to reviewers and researchers of the major problem to improvement of CRISPR-based antimicrobials are categorizing an efficient provision way ${ }^{\mathbf{5 2}}$.

\section{Conclusion:}

1. Genome-editing-technology is very important technique that empowers productions of the genetically-modified-cells \& organisms beings important to clarify gene role and human diseases mechanisms, And CRISPR/Cas9 system is one of Genome-editing-technology has been utilized for correcting DNA mutations that ranging from only single-base-pair into large deletion in the model systems of in vitro. \& in vivo..

2. This "CRISPR-Cas9 technology" uses a "ribonucleic acid (RNA)-guided deoxyribonucleic acid (DNA) endonuclease", Cas9, which induces "double-strand breaks (DSBs)" at target location, the DSBs. corrected by the use of a variety of cellular DNA-repair systems leading to changes in target sites, that technology have proven to be effective to investigation, prevention, and treatment of diseases.

3. The CRISPR-Cas9 technology has been quickly become a one of mainly important systems for genome-editing during essential biomedical investigate due to its adaptability \& simplicity.

4. The CRISPR Cas-9 technology have been given several benefits that accomplished the "zinc finger enzyme nuclease \& transcription activatorlike effector nuclease in human pluripotent stem cells \& stem cells of somatic origin"

5. Recently, researchers have been reveal a higher editing capability of the "transcription-activatorlike-effector-nuclease" in human stem cells is more efficiency about $79 \%$, of using for editing human iPSCs. Furthermore, CRISPR/Cas9 also utilized to monitor DNA noncoding sequences for recognizing the regulatory fundamentals to acknowledgment how the genetic variation were associated with cause the many of human diseases.

6. The CRISPR/Cas9 technology relates to editing efficiency "sgRNAs induce Cas9- mediated DSB" at favorite target position, "DSBs induces DNA-repair by HDRway"; conversely, an alternatives "DNArepair mechanism NHEJ" be able to induce at low frequencies, giving rise to unpredictable events of diminutive insertions $\&$ deletions.

7. Several recent studies have made clear importance of the CRISPR-Cas9 technology for the diagnosis and treatment to the many common diseases.

Acknowledgements: Not applicable.

Funding: None, Self-financing source

Availability of data and materials: Not applicable.

Ethics approval and consent to participate: Not applicable.

Patient consent for publication: Not applicable.

Competing Interests: All the authors declare that they have no competing interests.

\section{References}

1. Urnov FD, Miller JC, Lee YL, Beausejour CM, Rock JM and et al. Highly efficient endogenous human gene correction using designed zinc-finger nucleases. Nature, 2015; 435(2) : pp646-651

2. Bedell VM, Wang Y, Campbell JM, Poshusta TL, Starker CG, Krug 2nd RG, Tan W, and et al. In vivo genome editing using a high-efficiency TALEN system. Nature, 2012; 491: pp114-118.

3. Mali P, Esvelt KM and Church GM. Cas9 as a Versatile Tool for Engineering Biology. Nature Method, 2013; 10(10): p962

4. Razzaq A and Masood A. CRISPR/ Cas9 System: A Breakthrough in Genome Editing. Mol Biol., 2018; 7: p210. 
5. Travis T. Making the Cut: CRISPR GenomeEditing Technology Shows Its Power. Science, 2015; 350(6267): p1456.

6. Ledford H . CRISPR, the Disruptor. Nature, 2015; 522(7554): pp20-24.

7. Jinek, M, Chylinski K, Fonfara I, Hauer M, Doudna JA and Charpentier E. A programmable dual-RNAguided DNA endonuclease in adaptive bacterial immunity. Science,2012; 337(6096): pp816-821.

8. Marraffini LA. The CRISPR-Cas9 System of Streptococcus Pyogenes: Functionand Applications, Basic Biology to Clinical Manifestations Book. University of Oklahoma Health Sciences Center; 2016.

9. Maeder MLand Gersbach CA. Genome-editing Technologies for gene and cell therapy. Mol Ther.,2016; 24:pp430-446.

10. Rath D, Amlinger L, Rath A. and Lundgren M. The CRISPR-Cas immune system: Biology, mechanisms and applications. Biochimie.,2015; 117:pp $119-128$.

11. Charlesworth CT, Deshpande PS, Dever DP, Camarena J, Lemgart VT, Cromer MK and et al. Identification of preexisting adaptive immunity to Cas9 proteins in humans. Nat Med.,2019; 25:pp249-54.

12. Staahl BT, Benekareddy M, Coulon-Bainier C, Banfal AA, Floor SN, Sabo JK and et al. Efficient genome editing in the mouse brain by local delivery of engineered Cas 9 ribonucleoprotein complexes. Nat Biotechnol.,2017; 35:pp431-4.

13. Source: "What Is CRISPR-Cas9?," at http://www. yourgenome.org/facts/what-is-crispr-cas9.

14. Mali P, Yang L, Esvelt KM, Aach J, Guell M, DiCarlo JE, Norville JE, and Church GM. RNAguided human genome engineering via Cas9. Science, 2013; 339:pp 823-826.

15. Ran FA, Hsu PD, Wright J, Agarwala V, Scott DA, and Zhang F. Genome engineering using the CRISPR-Cas9 system. Nat. Protoc.,2013;8:pp 2281-2308.

16. Cong LF, Ran FA, Cox D. and et al. Multiplex genome engineering using CRISPR/Cas systems. Science,2013; 339(6121) pp819-823.

17. Jinek M, East A, Cheng, A, Lin S, Ma E. and Doudna J. RNA-programmed genome editing in human cells. eLife,2013; 2: p471.
18. Benedetta M, Motta and et al. The Impact of CRISPR/Cas9 Technology on Cardiac Research: From Disease Modelling to Therapeutic Approaches. Stem Cells International, 2017; 13 pages.

19. Ding Q, Regan, SN, Xia Y, Oostrom, LA, Cowan, CA, and Musunuru K. Enhanced efficiency of human pluripotent stem cell genome editing through replacing TALENs with CRISPRs. Cell Stem Cell, 2013; 12:pp 393-394.

20. Fulco CP, Munschauer M, Anyoha R and et al. Systematic mapping of functional enhancerpromoter connections with CRISPR interference. Science, 2016; 354(6313): pp769-773.

21. Sanjana NE., Wright J, Zheng K and et al. Highresolution interrogation of functional elements in the noncoding genome. Science,2016; 353(6307):pp 1545-1549.

22. Beaudoin M, Gupta RM, Won $\mathrm{HH}$ and et al. Myocardial infarction-associated SNP at 6p24 interferes with MEF2 binding and associates with PHACTR1 expression levels in human coronary arteries. Arteriosclerosis, Thrombosis, and Vascular Biology, 2015; 35(6) pp1472-1479.

23. Maruyama T, Dougan SK, Truttmann MC and et al. Increasing the efficiency of precise genome editing with CRISPR-Cas 9 by inhibition of nonhomologous end joining. Nature Biotechnology, 2015;33(5):pp. 538-542.

24. Hinson JT, Chopra A, Nafissi N and et al. HEART DISEASE. Titin mutations in iPS cells define sarcomere insufficiency as a cause of dilated cardiomyopathy, Science, 2015; 349(6251): pp 982-986.

25. Wang $\mathrm{Y}$, Liang $\mathrm{P}$, Lan $\mathrm{F}$ and et al. Genome editing of isogenic human induced pluripotent stem cells recapitulates long QT phenotype for drug testing. Journal of the American College of Cardiology,2014; 64(5): pp 451-459.

26. Zhang M, D'Aniello, C, Verkerk AO and et al. Recessive cardiac phenotypes in induced pluripotent stem cell models of Jervell and Lange-Nielsen syndrome: disease mechanisms and pharmacological rescue. Proceedings of the National Academy of Sciences of the United States of America, 2014; 111(50): pp5383-5392.

27. Wang X, Raghavan A, Chen T, Qiao L, Zhang Y, Ding Q and Musunuru K. CRISPR-Cas9 targeting 
of PCSK9 in human hepatocytes in vivo-brief report. Arterioscler Thromb Vasc Biol., 2016; 36:pp783-786.

28. Xie C, Zhang YP, Song L, Qi, W. and et.al. Genome editing with CRISPR/Cas9 in postnatal mice corrects PRKAG2 cardiac syndrome. Cell Res.,2016; 26:p1099.

29. Ma H, Marti-Gutierrez N, Park SW, Wu J, Lee Y and et al. Correction of a pathogenic gene mutation in human embryos. Nature, 2017;548:pp413-419.

30. Research LABlog, Gene Editing to Treat Diabetes, 2016; http://researchlablog.org/ post/144915391951/gene-editing-to-treat-diabetes.

31. American Diabetes Association, The Cost of Diabetes, http://www.diabetes.org/advocacy/newsevents/cost-of-diabetes.html.

32. Firth AL, Menon, T, Parker GS, Qualls SJ and et al. Functional gene correction for cystic fibrosis in lung epithelial cells generated from patient iPSCs. Cell Rep., 2015; 12:pp1385-1390.

33. Smith C, Abalde-Atristain L, He C, Brodsky BR, Braunstein EM, Chaudhari P, Jang YY and Cheng L. Efficient and allele-specific genome editing of disease loci in human iPSCs. Mol Ther., 2015; 23:pp570-577.

34. Kauf TL, Coates TD, Huazhi, L and et al. The Cost of Health Care for Children and Adults with Sickle Cell Disease. American Journal of Hematology, 2009; http://onlinelibrary.wiley.com/doi/10.1002/ ajh.21408/epdf.

35. Dever DP, Bak RO, Reinisch A, and et al. CRISPR/Cas9 beta-globin gene targeting in human haematopoietic stem cells. Nature, 2016; 539:PP384-389.

36. Canver MC, Smith EC, Sher, F; Pinello, L, Sanjana, NE, Shalem O, Chen, DD, and et al. BCL11A enhancer dissection by Cas9-mediated in situ saturating mutagenesis. Nature, 2015; 527:PP192197.

37. Yali L, Yang Yi, Xiangjin $\mathrm{K}$ and et al. One-step biallelic and scarless correction of a b-thalassemia mutation in patient-specific iPSCs without drug selection. Mol Ther Nucl Acids, 2017; 6:PP57-67.

38. Ohmori $\mathrm{T}$, Nagao $\mathrm{Y}$, Mizukami $\mathrm{H}$, and et al. CRISPR/Cas9-mediated genome editing via postnatal administration of AAV vector cures haemophilia B mice. Sci Rep., 2017; 7:P4159.
39. Park CY, Kim, DH, Son JS, Sung JJ, Lee J, Bae S. Functional correction of large factor VIII gene chromosomal inversions in hemophilia A patientderived iPSCs using CRISPR-Cas9. Cell Stem Cell, 2015; 17:pp213-220.

40. Chang, CW, Lai YS, Westin E, KhodadadiJamayran A, and et al. Modeling human severe combined immunodeficiency and correction by CRISPR/Cas9-enhanced gene targeting. Cell Rep.,2015; 12:pp 1668-1677.

41. De Ravin SS, Li, L, Wu, X, Choi, U, Allen C and et al. CRISPRCas9 gene repair of hematopoietic stem cells from patients with X-linked chronic granulomatous disease. Sci Transl Med., 2017; 9:p3480.

42. Jing $W$, Zhang $X$, Sun, $W$, Hou, $X$, Yao $Z$ and Zhu Y. CRISPR/CAS9-mediated genome editing of miRNA-155 inhibits proinflammatory cytokine production by RAW264.7 cells. Biomed Res Int., 2015:pp326-042..

43. Cwetsch, A.W., Pinto, B.; Savardi, A.; Cancedda, L. In vivo Method for acute modulation of gene expression in the central nervous system. Prog. Neurobiol.,2018; 168:pp69-85.

44. McKinnon PJ. DNA repair deficiency and neurological disease. Nat Rev Neurosci.,2009;10:pp100-12.

45. Monteys AM, Ebanks SA, Keiser MS and Davidson BL. CRISPR/Cas9 editing of the mutant huntingtin allele in vitro and in vivo. Mol Ther., 2017; 25:pp12-23.

46. Jamie T. News from the Society for Neuroscience Annual Meeting: gene editing techniques show promise in silencing or inhibiting the mutant Huntington's disease gene. Neurol Today, 2015;15:pp14-16.

47. Ouellet DL, Cherif K, Rousseau J, Tremblay JP. Deletion of the GAA repeats from the human frataxin gene using the CRISPR-Cas9 system in YG8R-derived cells and mouse models of Friedreich ataxia. Gene Ther.,2017; 24:pp265-274.

48. Wang L, Yi F, Lina, F, Yang J and et al. CRISPR/ Cas9-mediated targeted gene correction in amyotrophic lateral sclerosis patient iPSCs. Protein Cell, 2017;8:pp365-378.

49. Hammond A, Galizi R and Kyrou K. A CRISPRCas9 Gene Drive System Targeting Female Reproduction in the Malaria Mosquito Vector 
Anopheles gambiae. Nature Biotechnology,2016; 34: pp. 78-83.

50. Adler J. Kill All the Mosquitoes?. Smithsonian Magazine, 2016; http://www.smithsonianmag.com/ innovation/kill-all-mosquitos-180959069/?no-ist).

51. Centers for Disease Control and Prevention.
Antibiotic Resistance Threats in the United States, 2013; https://www.cdc.gov/drugresistance/threatreport-2013/index.html.

52. Barrangou $\mathrm{R}$ and Doudna JA. Applications of CRISPR Technologies in Research and Beyond. Nature Biotechnology, 2016; 34(9) p937. 